\title{
Arsenic methylation and its relationship to abundance and diversity of arsM genes in composting manure
}

\author{
W.W. Zhai, X.J. Tang \& J.M. Xu \\ Institute of Soil and Water Resources and Environmental Science, College of Environmental and Resource Sciences, \\ Zhejiang Provincial Key Laboratory of Agricultural Resources and Environment, Zhejiang University, Hangzhou, \\ P.R. China
}

\begin{abstract}
In this study, two pilot-scale pig manure composting piles were constructed for a systematic investigation of arsenic (As) methylation during manure composting. Microbial community composition, as well as the abundance and diversity of arsM genes were monitored using real-time PCR (qPCR) and amplicon sequencing of both 16S rRNA and arsM genes. Results show an overall accumulation of methylated As occurring during 60 day-composting time. The arsM gene copies increased gradually over time and were correlated positively to the concentrations of methylated As. 16S rRNA gene sequencing and arsM clone library analysis confirmed that the high abundance and diversity of arsM genes shared the same known As-methylating microbes, including Streptomyces sp., Amycolatopsis mediterranei and Sphaerobacter thermophiles, which were likely involved in the methylation process. These results demonstrated that As methylation during manure composting is significant. For the first time, the linkage between As biomethylation and the abundance and diversity of the arsM functional gene in composting manure was established.
\end{abstract}

\section{INTRODUCTION}

Arsenic (As)-based feed additives are commonly used in the poultry and livestock industry. Not readily absorbed in animal tissues, almost all the fed As is excreted without attenuation in manure at concentrations up to $300 \mathrm{mg} \mathrm{kg}^{-1}$ (Kiranmayi et al., 2015). Methylation of As is normally regarded as one of the main detoxification pathways for As in environment, which is catalyzed by S-adenosylmethionine methyltransferase encoded by arsM genes (Qin et al., 2006). Although the mechanism of microbial As methylation is known and arsM genes have been detected in various environments, there remains a limited understanding of how the abundance and diversity of arsM genes correlate with the methylation process during manure composting.

\section{METHODS/EXPERIMENTAL}

\subsection{Composting experiments and sampling}

Two independent pilot-scale $(2.5 \mathrm{~m} \times 1.8 \mathrm{~m} \times 0.75 \mathrm{~m}$ in length, width and height) pig manure compost piles were set up in Hangzhou, China, containing on average $1750 \pm 60 \mu \mathrm{g} \mathrm{kg}^{-1} \mathrm{As}(\mathrm{dw}, \mathrm{n}=3)$. The first manure compost pile (MC1) was composed of $1,200 \mathrm{~kg}$ pig manure and $600 \mathrm{~kg}$ sawdust for optimal $\mathrm{C} / \mathrm{N}$ ratio and water content. The second manure compost pile (MC2) contained $1,200 \mathrm{~kg}$ pig manure and $600 \mathrm{~kg}$ sawdust mixed with burned rice straw. The moisture contents of the composting sites were maintained at approximately $65 \%$ by sprinkling water once every two days.
The compost piles were turned over and mixed once every two days in the first month, and once every four days in the second month for aeration. The whole composting process lasted for $60 \mathrm{~d}$, and $2 \mathrm{~kg}$ samples were collected on day $1,5,15,25,35,45$ and 60 .

\subsection{Analysis method}

Concentrations of As species were measured by High-Performance Liquid Chromatography Coupled with Inductively Coupled Plasma Mass Spectrometry (HPLC-ICP-MS, NEXION300XX, PerkinElmer, Inc., USA). Total DNA was extracted and copy numbers of arsM gene in the compost samples were estimated by qPCR. 16S rRNA gene was amplified, then sent for sequencing using Illumina Miseq sequencing platform (Miseq, Illumina Inc., USA). Four samples (15-MC1, 15-MC2, 60-MC1 and 60-MC2) were selected for the construction of ars $M$ gene clone libraries. The detail were described elsewhere (Zhai et al., 2017).

\section{RESULTS AND DISCUSSION}

\subsection{The change of As species during composting}

The total concentrations of methylated As species increased more rapidly during the mesophilic and thermophilic phases, while only small increases during the maturing phase. The concentrations of methylated As species represented 37\% (MC1) and 35\% (MC2) of total As concentration by day 60, clearly indicating As methylation during manure composting. Methylated As species analysis also showed that MMA 


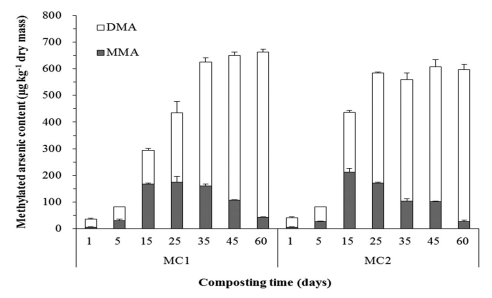

Figure 1. Changes in concentrations of methylated As (MMA and DMA) in the two compost piles.

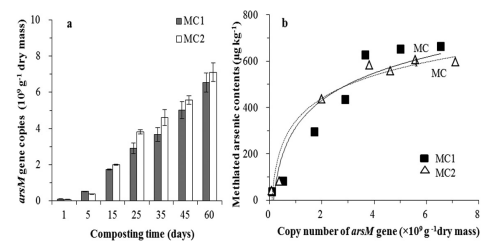

Figure 2. Plot of arsM gene copies (a) and plot of methylated As concentration versus arsM copies in two compost piles (b).

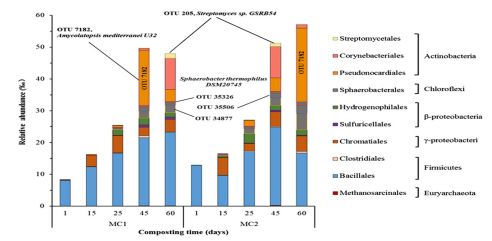

Figure 3. Changes and taxa of selected 16S OTUs related to As methylation.

content in both compost piles peaked during the thermophilic phase (day 5-42) and dropped rapidly during the maturing phase (day 43-60). In contrast, DMA concentrations increased steadily in both compost piles over the composting period (Fig. 1), indicating the transformation of MMA to DMA. And the conversion of DMA to TMA is the rate limiting step in As methylation. Therefore, DMA commonly accumulates in environment samples.

\subsection{Copy numbers of bacterial arsM genes}

The abundance of arsM genes in MC1 and MC2 as a function of composting time increased gradually from $\sim 0.1 \times 10^{9}$ to $\sim 6.8 \times 10^{9}$ copies g-1 dry mass in both piles (Fig. 2a). Further, the sum of MMA and DMA concentrations at different time points was found to correlate strongly with the ars $M$ gene copy numbers (Fig. 2b). Considering the arsM gene is the key functional gene responsible for microbial As methylation, the positive relationship between the concentrations of methylated As and the copy numbers of arsM genes confirmed the As methylation ability in the manure compost piles.

\subsection{Abundance and biodiversity of ars $M$ genes}

We aligned the 16S rRNA sequences from the compost piles against $16 \mathrm{~S}$ rRNA sequences from microbes

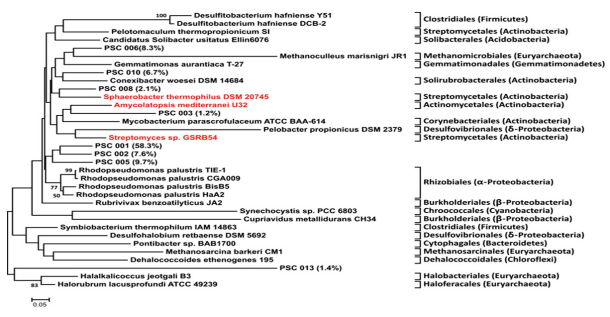

Figure 4. Neighbor-joining analyses of arsM sequences retrieved from composting samples.

containing an arsM gene. Eighty-three OTUs were identified as matching (similarity $\geq 95 \%$ ) and their relative abundances clearly increased with composting time (Fig. 3). A Neighbor-joining tree of the 8 most abundant ars $M$ PSCs ( $>1 \%$ relative abundance) was constructed with selected references. Phylogenetic analysis of arsM clone showed the high diversity of arsM gene in the composting samples (Fig. 4). The present study showed putative arsM affiliated with a wide range of phylogenetic taxa that were present in all the composting samples.

\section{CONCLUSIONS}

In conclusion, two pilot-scale pig manure compost piles were established and an accumulation of methylated As was revealed over the composting process. By qPCR, 16S rRNA sequencing and clone libraries, an increasing in abundance and diversity of arsM genes in composting pig manure were confirmed.

\section{ACKNOWLEDGEMENTS}

This work was financially supported by the Provincial Public Technology and Applied Research Projects by Science and Technology Department of Zhejiang Province (2014C33020), the National Key Technology Research and Development Program of the Ministry of Science and Technology of China (2014BAD14B04), Zhejiang Provincial Natural Science Foundation of China (LR13D010001) and Fundamental Research Funds for the Central Universities.

\section{REFERENCES}

Kiranmayi, P. M., Asok, A. \& Lee, B. 2015. Organoarsenicals in poultry litter: Detection, fate, and toxicity. Environ. Int. 75: 68-80.

Qin, J., Rosen, B. P., Zhang, Y., Wang, G.J., Franke, S., \& Rensing, C. 2006. Arsenic detoxification and evolution of trimethylarsine gas by a microbial arsenite Sadenosylmethionine methyltransferase. Proc. Natl. Acad. Sci. U.S.A. 103(7): 2075-2080.

Zhai, W.W., Wong, M.T., Luo, F., Hashmi, M.Z., Liu, X.M., Edwards, E.A., Tang, X.J. \& Xu, J.M. 2017. Arsenic methylation and its relationship to abundance and diversity of arsM genes in composting manure. Sci. Rep. 7: 42198. 